\title{
A possible role of astrocytes in contextual memory retrieval: An analysis obtained using a quantitative framework
}

\author{
Shivendra Tewari ${ }^{1,2 *}$ and Vladimir Parpura ${ }^{3,4 *}$ \\ 1 Biotechnology and Bioengineering Center, Medical College of Wisconsin, Milwaukee, WI, USA \\ ${ }^{2}$ Department of Physiology, Medical College of Wisconsin, Milwaukee, WI, USA \\ ${ }^{3}$ Department of Neurobiology, University of Alabama at Birmingham, Birmingham, AL, USA \\ ${ }^{4}$ Department of Biotechnology, University of Rijeka, Rijeka, Croatia
}

\section{Edited by:}

Gabriel A. Silva, University of

California, San Diego, USA

Reviewed by:

Gabriel A. Silva, University of

California, San Diego, USA

Massoud L. Khraiche, University of

California San Diego, USA

\section{*Correspondence:}

Shivendra Tewari, Molecular \&

Integrative Physiology, University of

Michigan, NCRC Building 10 \#A122,

2800 Plymouth Rd, Ann Arbor,

MI 48109, USA

e-mail: tewarisg@gmail.com;

Vladimir Parpura, Department of

Neurobiology, University of Alabama

at Birmingham, 1719 6th Avenue

South, CIRC 429, Birmingham,

AL 35294, USA

e-mail:vlad@uab.edu

\section{INTRODUCTION}

One of the most intriguing properties of the brain is to learn and form new memories. Memories are essential for guiding effective actions, for understanding the words we read, for recognizing the objects we see, etc. It is well established that retrieval of a particular memory is facilitated when the context at the time of memory retrieval is similar to that of the initial learning experience (Maren and Holt, 2000). This phenomenon, termed as context-dependent memory, also provides an explanation as to why memory recall is impaired when people, place or things are experienced "out of context." The process by which the context is embedded into learning of information, and can later facilitate memory retrieval is known as contextual memory retrieval (Maren and Holt, 2000). Pavlovian conditioning, i.e., classical conditioning, is an exemplar form of contextual fear memory learning and retrieval (Maren, 2001).

Ever since memory deficits, initially characterized in patients PB and FC (Milner and Penfield, 1955) and later HM (Scoville and Milner, 1957), were associated with hippocampus damage, it has become clear that the hippocampal region plays a crucial role in the acquisition of new semantic and episodic memory (Andersen et al., 2007); but see (Corkin, 2002). Apart from storing memories, the appropriate retrieval of stored memories is also of paramount importance for maintaining relationships, achieving personal and professional goals, and carrying out even simple day to day activities. The critical role of the hippocampus in memory retrieval has gone largely uncontested and has been supported by various studies including lesion studies (Holt and Maren, 1999), unit studies (Paller and McCarthy, 2002), as well as functional neuroimaging (Eldridge et al., 2000).

Lee and Kesner (2004) reported a sub-region specific contribution of the hippocampus in memory acquisition and retrieval. Interpretation of the result suggests that the CAl region is uniquely associated with a reliable retrieval of the contextual memory. Such a role for the CA1 region in contextual memory retrieval has also been highlighted in several studies (Hall et al., 2001; Khuchua et al., 2003; Murchison et al., 2011). Previous work suggests that the firing synchrony in neighboring CA1 pyramidal neurons might be a putative mechanism of contextual memory retrieval in the CA1 region (Hall et al., 2001; Siekmeier et al., 2007; Takahashi and Sakurai, 2009). However, data are accumulating suggesting that the coordination between neurons and astrocytes may play a role in information processing and memory retrieval.

Fellin et al. (2004) demonstrated that the activation of $\mathrm{Ca}^{2+}$ elevation in astrocytes, by various stimuli, produces slow inward currents (SICs) in 2-12 CA1 pyramidal neurons, synchronously. They found that repetitive stimulation of astrocytes leads to synchronous firing of the same CA1 pyramidal neurons, mainly due to the activation of neuronal NR1/NR2B subunits of the extrasynaptic N-Methyl-D-aspartate receptors (eNMDARs). It should be noted that NMDAR NR2B subtype plays a crucial role in 
encoding and retrieval of contextual fear memory (Zhao et al., 2005; Zhang et al., 2008; Zhuo, 2009); but see Nakazawa et al. (2004). Thus, it appears that the interaction/signaling between neurons and astrocytes plays a role in information processing and memory retrieval.

Astrocytes in the hippocampus have numerous highly branched fine processes that extend to distances up to $100 \mu \mathrm{m}$ and enwrap asymmetrical (mainly excitatory/glutamatergic) synapses of a relatively small group of neurons (Fellin et al., 2006); of note, individual astrocytic domains do not overlap (Bushong et al., 2002, 2004; Ogata and Kosaka, 2002; Nedergaard et al., 2003; Oberheim et al., 2009). Thus, strategically, astrocytes are ideally positioned to sample electrical activity patterns of the Schaffer collateral input from CA3 pyramidal neurons and retrieve their associated context in neighboring CA1 pyramidal neurons. During the retrieval, the firing pattern of the pyramidal neurons, in the CA3-CA1 region, is quite similar to that recorded during memory formation (Small et al., 2001). As astrocytes play an important role in short-term and long-term potentiation of individual synapses (Martin et al., 2007; Henneberger et al., 2010), it is possible that they also contribute to various aspects of memory. We propose that astrocytes facilitate the memory storage and retrieval by sensing encoding/retrieval patterns of neurons that reside within individual astrocytic domains and, in turn, astrocytes signal the associated CA1 pyramidal neurons.

Based on these recent experimental observations, we developed a computational framework/model, where an astrocyte modulates synchrony among CA1 pyramidal neurons within a domain of $100 \mu \mathrm{m}$ in diameter, which qualitatively reproduces the biological experimental findings (Fellin et al., 2004). Power spectral density analysis of the computed local field potentials indicates a shift in neuronal firing rhythm from broad-frequency range in the absence of astrocytic gliotransmission to a deltadominant rhythm when gliotransmission is turned on. As delta rhythm has been implicated in cognitive domain (Knyazev, 2012) and consolidation of declarative memories (Ruch et al., 2012), this might be a putative mechanism of memory retrieval tuned by astrocytes, which will require further experimental verification in biological preparations. Apart from a possible role in memory retrieval, using the computational model, we propose that astrocytes may lead to epileptiform discharges in CA1 pyramidal neurons, as has been previously demonstrated in biological preparations (Kang et al., 2005; Tian et al., 2005). Such pathological discharges occuring under computational conditions warrant an increase in eNMDAR plasmalemmal activity that could arise from an overexpression of such receptors and/or positive modulation of their conductance (Omkumar et al., 1996; Liao et al., 2001).

\section{MATERIALS AND METHODS OVERVIEW OF THE MODEL ARCHITECTURE}

The complete network (shown in Figure 1) comprises of three major components: a CA3 pyramidal neuron, four CA1 pyramidal neurons and an astrocyte in the stratum oriens (s.o.). A CA3 pyramidal neuron is computationally described by Traub's branching dendrite model of the CA3 pyramidal neuron comprising of 21 compartments (Traub et al., 1994). Each of the

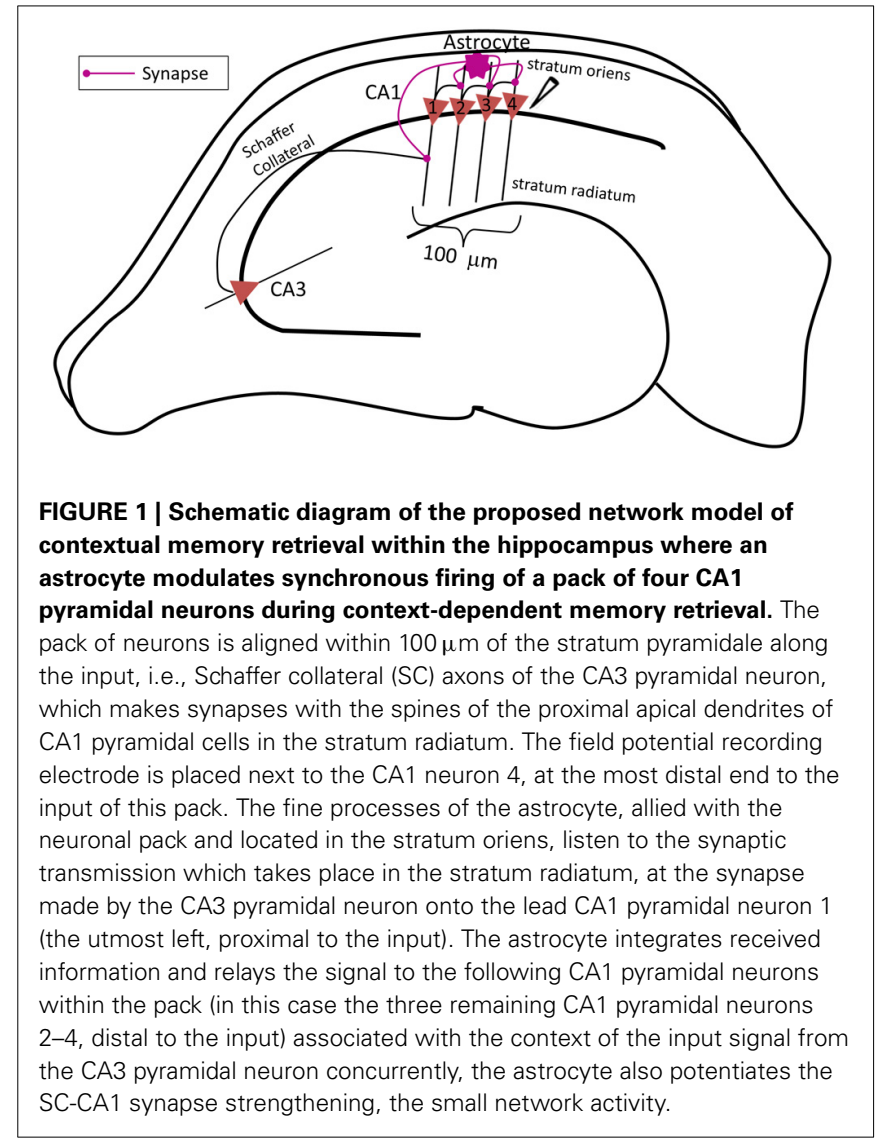

four CA1 pyramidal neurons is described by the 19-compartment CA1 pyramidal neuron model (Traub et al., 1991), which was modified to incorporate four additional compartments accounting for the axon initial segment (IS), axon proper, and two spines (placed at the $6^{\text {th }}$ and $12^{\text {th }}$ compartment of the original CA1 pyramidal neuron model) to yield a 23-compartment CA1 pyramidal neuron model. Axon and spine compartments were added to form CA1-CA1 synapses in the s.o. at the basal dendrites. The dendritic spine model utilized here is from Tewari and Majumdar (Tewari and Majumdar, 2012a), but was modified to include synaptic NMDARs and eNMDARs. The astrocyte model is also adopted from Tewari and Majumdar (Tewari and Majumdar, 2012a,b), and it accounts for gliotransmitter (glutamate) release/astrocyte-dependent SICs through the activation of $\mathrm{NR} 2 \mathrm{~B}$-containing receptors on the CA1 neurons. It is assumed that the CA3 pyramidal neuron makes 25 synapses with the CA1 neuron 1 (Figure 1). The number of synapses at the CA1-CA1 synapses is assumed to be 20 . These numbers were obtained by a hit and trial approach, and reflect the smallest numbers possible to have a successful signal transduction of the proposed network. All associated model equations and parameters are provided in the supplementary materials.

As shown in Figure 1, the CA3 neuron forms synapses with the CA1 neuron 1, at the spines in the stratum radiatum (s.r.). To model glutamate release from the CA 3 and CA1 neurons, we use a continuous function that transforms axon voltage into glutamate 
concentration in the cleft (Destexhe et al., 1994):

$$
[g]=\frac{g_{\max }}{1+\exp \left[-\left(V_{\text {axon }}-V_{\mathrm{p}}\right) / K_{\mathrm{p}}\right]},
$$

where $g_{\max }$ is the maximum concentration of glutamate in the synaptic cleft, $V_{\text {axon }}$ is the membrane potential in the axon compartment of the glutamate releasing neuron, $K_{\mathrm{p}}$ gives the steepness, and $V_{\mathrm{p}}$ is the axon voltage at which glutamate concentration is half of the maximum (see Table 1). The glutamate released from CA3 neuron is sensed by the astrocyte in the s.o. (Perea and Araque, 2005) leading to astrocytic $\mathrm{Ca}^{2+}$ elevation. Simultaneously, glutamate released from the $\mathrm{CA} 3$ neuron leads to an inward current in the CA1 neuron 1. The SICs evoked by the astrocyte through eNMDARs are:

$$
I_{\mathrm{SIC}}=g_{\mathrm{NR} 2 \mathrm{~B}} \cdot h \cdot v_{\mathrm{s}},
$$

where $g_{\mathrm{NR} 2 \mathrm{~B}}$ is the conductance through NR1/NR2B-containing receptors, $v_{\mathrm{s}}$ is the spine membrane potential and $h$ is the gating variable of eNMDAR that depends on the astrocyte as described by the following equation:

$$
\frac{d h}{d t}=\frac{\sigma}{\tau_{1}}-\frac{h}{\tau_{2}} .
$$

The above relation is regulated by astrocytic $\mathrm{Ca}^{2+}$ by $\sigma$, which has a value of 0.4 (representing the maximum open probability of eNMDAR) whenever astrocytic $\mathrm{Ca}^{2+}$ goes above $200 \mathrm{nM}$ (Parpura and Haydon, 2000; Nadkarni and Jung, 2003; Tewari and Majumdar, 2012b), otherwise set to zero. The time constants $\tau_{1}$ and $\tau_{2}$ govern the rise-time and decay-time of the eNMDAR currents, and are based on the experimental observation (Fellin et al., 2004); parameter values are listed in Table 1.

A given CA1 neuron forms synapses with the other CA1 neurons in the basal dendrite region (Andersen et al., 2007); in the proposed network model, the synapses are formed at the $6^{\text {th }}$ compartment of the CA1 neuron model. The sequence in which the CA1 neurons make synapses is as follows: $1 \rightarrow 2,2 \rightarrow 3,3 \rightarrow 4$ (Figure 1, left to right); the CA1 neuron 4 is not connected to any other neuron. The astrocyte senses the signal (glutamate) released by the CA3 neuron and potentiates the above CA1 neuron synapses by releasing gliotransmitter (glutamate) in the vicinity of the eNMDARs on the spines, which leads to synchronized firing of the CA1 neurons. The astrocyte releases glutamate from

Table 1 | List of parameters involved at the synapses.

\begin{tabular}{lll}
\hline Symbol & Value & Reference \\
\hline$g_{\max }$ & $3.5-4 \mathrm{mM}$ & Danbolt, 2001 \\
$V_{p}$ & $72 \mathrm{mV}^{\dagger}$ & Destexhe et al., 1994 \\
$K_{p}$ & $5 \mathrm{mV}$ & Destexhe et al., 1994 \\
$g_{\mathrm{NR} 2 \mathrm{~B}}$ & $0.6 \mathrm{nS}$ & Gasparini et al., 2004 \\
$\tau_{1}$ & $92.3 \mathrm{~ms}$ & Fellin et al., 2004 \\
$\tau_{2}$ & $568.5 \mathrm{~ms}$ & Fellin et al., 2004 \\
\hline
\end{tabular}

${ }^{t}$ This value is reported with respect to the resting membrane potential. glutamate secretory vesicles utilizing $\mathrm{Ca}^{2+}$-dependent exocytosis as soon as astrocytic $\mathrm{Ca}^{2+}$ concentration exceeds the threshold $\mathrm{Ca}^{2+}$ concentration for triggering secretion [see (Tewari and Majumdar, 2012a) for details].

\section{COMPUTER SIMULATIONS}

The proposed network model invoked a total of 1247 ordinary differential equations which were solved using a quasi-constant step sized backward-difference numerical solver implemented in MATLAB (The MathWorks, Natick, MA). The system of ordinary differential equations was solved for a duration of $50 \mathrm{~s}$ (model time) which took approximately $8 \mathrm{~h}$ (real time) to compute.

\section{LOCAL FIELD POTENTIAL CALCULATIONS}

The somata of the CA1 neurons were spaced $25 \mu \mathrm{m}$ apart. The recording electrode was placed $25 \mu \mathrm{m}$ away from the soma of the CA1 neuron 4 (see Figure 1). The local field potential was calculated using line source approximation (Gold et al., 2006). Based on the ionic currents generated from the network simulation (in the presence and absence of the astrocyte), trans-membrane currents across each compartment were calculated for each of the CA1 neurons. Please note that the contribution from IS and axon compartments was neglected because myelinated axon and nodes of Ranvier make no contribution to the local field potential (Gold et al., 2006). Afterwards, linear source approximation was used to compute local potentials at the recording electrode using a custom-written MATLAB program. Mathematically, field potential is calculated using the following expression:

$$
\Phi(t)=\sum_{\forall c} \frac{\rho_{\mathrm{c}} I_{\mathrm{c}}}{4 \pi r_{\mathrm{c}}},
$$

where $I_{\mathrm{c}}$ is the trans-membrane current across compartment $\mathrm{c}$, $r_{\mathrm{c}}$ is the distance of compartment $\mathrm{c}$ from the recording electrode, and $\rho_{\mathrm{c}}$ is the extracellular resistivity at compartment $\mathrm{c}$. The value of $\rho_{\mathrm{c}}$ for soma and proximal apical dendritic compartments is $643 \Omega \mathrm{cm}$ (Lopez-Aguado et al., 2001), while for the basal and distal apical dendritic compartments it is 260 and $287 \Omega \mathrm{cm}$, respectively (Lopez-Aguado et al., 2001).

\section{RESULTS}

\section{NETWORK SIMULATIONS}

The network is simulated in the absence and in the presence of astrocytic activity, i.e., a feedback to neurons in respect to synaptic transmission. Figure 2 shows representative action potential (AP) bursts in the axon compartment of the CA3 neuron that lead to subsequent firing in the CA1 neurons $1-4$, with a slight delay, as expected from the flow of the input signal in the CA1 region. The network was then simulated again, while incorporating the astrocytic response to neuronal synaptic transition, i.e., gliotransmission, that leads to an induction of SICs in the CA1 neurons. We show a representative astrocytic response and its associated effects on the firing of the CA1 neurons (Figure 3). It is apparent by comparing the two different simulations (compare Figures 2 and 3; and Figures 4A and 4B) that the astrocyte makes the CA1 neurons to fire in synchrony, and in a different pattern. Note that the astrocyte induces SIC twice (data not shown; this effect is 
captured by the gating variable $h$ in Equation 3) while the astrocytic $\mathrm{Ca}^{2+}$ concentration is above the threshold for triggering exocytotic release of glutamate (see the shaded area in Figure 3).

\section{LOCAL FIELD POTENTIALS}

Local field potential (LFP) calculation at the point of interest is based on linear source approximation in the absence and presence of the astrocyte. It is apparent from the peaks of LFP that the CA1 neurons fire synchronously under the astrocyte-dependent pathway (Figures 4 and 5). Hence, after the addition of the astrocyte-induced SICs, the CA1 neuron pack starts firing at a different rate, which is dependent on astrocyte $\mathrm{Ca}^{2+}$ oscillations
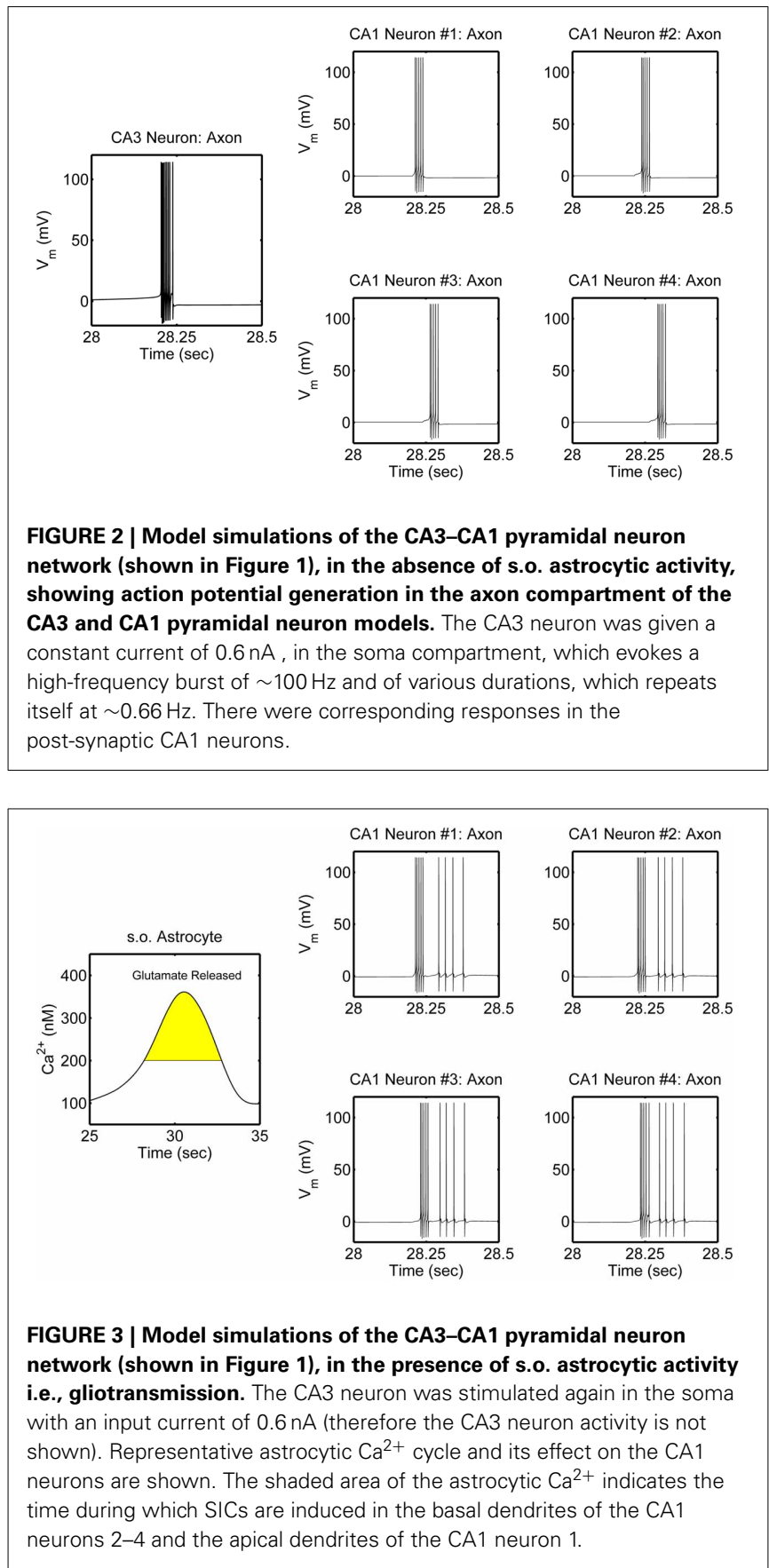

(Figure 4). The LFP duration is much shorter in the absence of astrocytic activity (Figure 5A) compared to the LFP duration in the presence of an active astrocyte (Figure 5B); the input signal, from the CA3 neuron, was same for both pathways. To assess a possible difference in the dominant frequency between the two pathways, we used Fast Fourier Transform (FFT) spectrum analysis (Figures 5D,E). Based on this analysis, the astrocyteindependent pathway has wide-spread activity with multiple peaks, a pattern likely resulting due to the differences in the firing rate of each CA1 neuron within the pack; the main proportion of the activity forms an envelope, around $4-11 \mathrm{~Hz}$, lacking any typical rhythm (see Figure 5D). The FFT analysis of the astrocytedependent pathway shows a shift towards lower frequency with

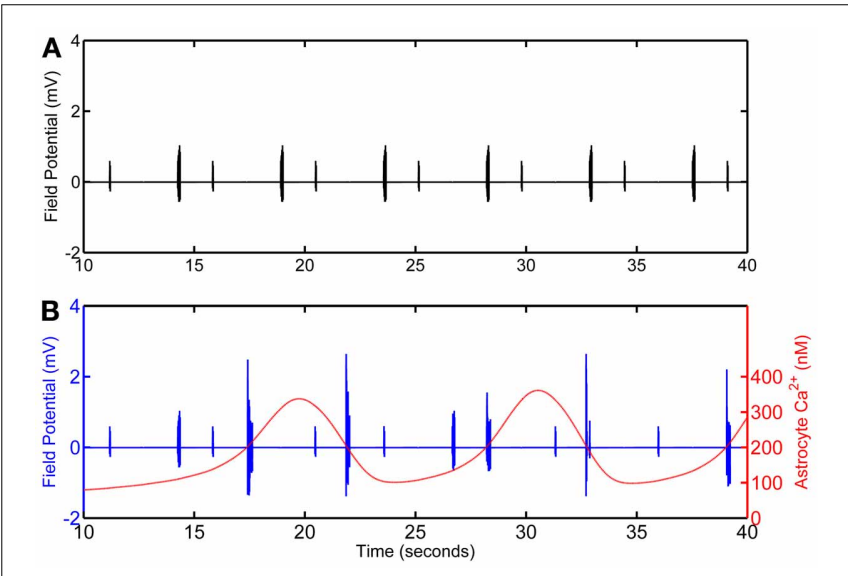

FIGURE 4 | Calculated field potential based on model simulations of the network in the absence (A) and in the presence (B) of the astrocyte. The simulation omits the first $10 \mathrm{~s}$ of simulation as, in the model, astrocytic $\mathrm{Ca}^{2+}$ oscillations begin after $10 \mathrm{~s}$ of the CA3 neuronal activity.
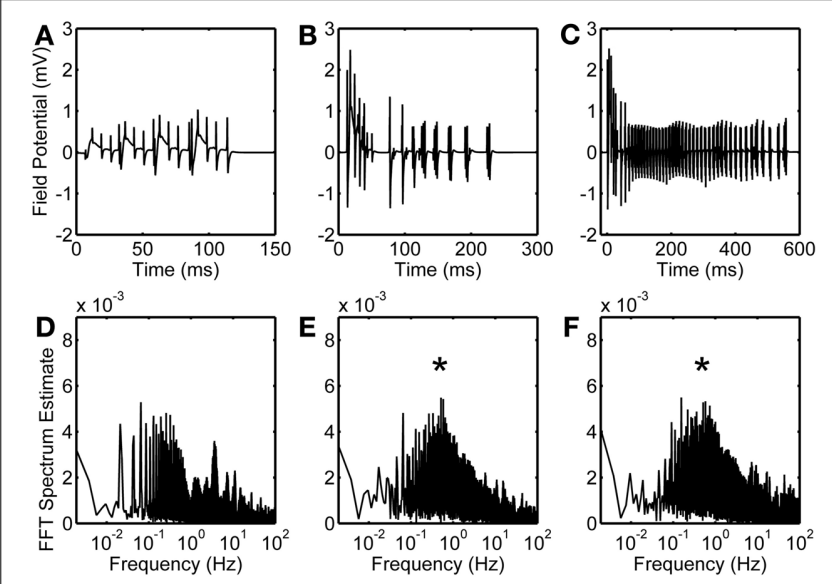

FIGURE 5 | Astrocytic contribution to field potential recordings. Duration of evoked field potentials in the absence of astrocyte (A), in the presence of astrocyte activity (B), and in the presence of astrocytic activity along with an enhanced eNMDAR conductance in CA1 neurons (C). Corresponding FFT spectra are shown in (D,E, and $\mathbf{F})$, respectively. Note that FFT spectra are calculated based on complete simulated data of $50 \mathrm{~s}$ Asterisks denote the effect of astrocyte-induced SICs. 
sharpened tuning, resulting in an occurrence of a delta rhythm (Figure 5E, asterisk), possibly due to synchronous firing of the CA1 neurons in tune with the astrocyte gliotransmission.

\section{EPILEPTIFORM SIMULATIONS}

NMDARs are known to play a role in the initiation and propagation of epileptiform discharges (Auzmendi et al., 2009). To study the possible effect of glutamatergic gliotransmission on the eNMDARs, as demonstrated in biological preparations [e.g., (Fellin et al., 2004)], we simulated the astrocyte-dependent pathway after doubling the eNMDAR conductance in the CA1 neurons (Table 1). This computational alteration emulates a biological condition of an increased presence of plasmalemmal eNMDARs (due to an overexpression/increased trafficking) and/or flux through an unaltered number of channels. As a control, we simulated the same network under conditions incapacitating gliotransmission of the s.o. astrocyte, caused by clamping astrocytic $\mathrm{Ca}^{2+}$ at $100 \mathrm{nM}$, which is below the threshold for glutamate release. The simulation data suggest that the presence of the astrocyte lacking gliotransmission does not affect the firing of the CA1 neurons overexpressing eNMDARs upon their stimulation by the CA3 neuron (compare Figure $\mathrm{S} 1$ and Figure 2). However, the astrocytic activity/gliotransmission may induce epileptiform discharges in the CA1 neurons via an increased eNMDAR activity, as evidenced by the grossly exaggerated duration of LFPs (Figure 5C). FFT spectrum analysis of the epileptiform activity further indicates that the astrocyte activity tunes the CA1 neurons to fire in a delta rhythm, while other surrounding frequencies appeared suppressed (Figure 5F, asterisk).

\section{DISCUSSION}

Herein we presented a biophysically detailed mathematical framework of the CA3/Schaffer collateral-CA1 pyramidal neuron signal transduction that is based on experimental reports suggesting a possible role of astrocytes in contextual memory retrieval by synchronizing CA1 pyramidal neuronal network and achieving a delta rhythm. We were interested in studying a small neuronal network for two reasons: (i) a small network model can be kept biophysically detailed, and (ii) to overcome the experimental limitations in biological preparations (i.e., a lack of the spatial resolution to record field potentials from such a small network, as opposed to recording from a much larger area in reality). We did analysis of the simulated local field potentials generated in the absence and in the presence of astrocytic activity. The results suggest that astrocytic gliotransmission potentiates the synapses through eNMDARs, synchronizes CA1 neurons and modulates their overall firing rate. Frequency domain analysis of the simulated LFP suggests that astrocytic $\mathrm{Ca}^{2+}$ signaling shifts the neuronal activity in the CA1 region from a broad spectrum frequency lacking any typical rhythm towards a delta frequency. Interestingly, a recent study (Grover et al., 2009) described the induction of long-term potentiation in the CA1 region due to a burst stimulation over a broad frequency range centered around delta frequency, albeit the underlying mechanism was not investigated. Thus, our in silico model offers a possible computational explanation to this delta frequency and it should be additionally explored in biological preparations.
Delta rhythm is a low frequency brain activity which is implicated in cognitive domain such as attention, salience detection, and subliminal perception (Knyazev, 2012). Moreover, it is known that sensory cues innervate the CA1 pyramidal neurons associated with the context. Under such circumstances repetition of an item stimulus will recover the contextual state, in the entorhinal cortex, in response to firing of the associated pyramidal cells in the CA1 region. In our circuitry model, we observe that astrocytic input, which makes CA1 pyramidal neurons to fire in a synchronous manner, eliminates group firing activity at other frequencies except delta, suggesting a possible role of astrocytes in memory retrieval. From a functional point of view, this finding proposes a cognitive role of astrocytes where an environmental cue demands attention, e.g., delta rhythm generation in visual cortex depends on the activity to which attention is paid (Lakatos et al., 2008). The model suggests that similar activity of delta rhythm generation might exist in the CA1 region of hippocampus during events of contextual memory retrieval.

NR2B subunit overexpression enhances chronic pain in mice (Zhuo, 2009). Additionally, NR2B-containing NMDARs are linked to cell death and seizures (Ding et al., 2007). To test the functional consequence of NR2B overexpression on CA1 neuronal activity, we increased the conductance of NR2B-containing NMDARs which is equivalent to an insertion of eNMDARs in the plasmalemma or enhanced unitary conductance of the eNMDARs. Such a condition simulates an increased access of astrocytic transmission to the NR2B-containing NMDARs. The results suggest that enhanced conductance of NR2B-containing NMDARs leads to a high-frequency oscillation of field potentials. The duration of these field potentials is $\sim 600 \mathrm{~ms}$ which is equal to the duration of SIC via NR2B-containing NMDARs. This activity could be a molecular correlate of seizures seen in the hippocampus, which are sensitive to NR2B subunit antagonists (Kolarova et al., 2003; Butler et al., 2010).

In our present work, we have not adjusted our model for micro-anatomical restructuring at the tripartite synapse, which would almost inevitably occur due to the dynamics at astrocytic filopodia/perisynaptic processes (Hirrlinger et al., 2004; Lavialle et al., 2011). Such restructuring could lead to changes in the proximity of neuronal and astrocytic release and/or input sites. Future dynamic modeling to simulate micro-anatomical arrangements would be needed to computationally address the role that astrocytic structural plasticity may play in tuning neuronal firing rhythm.

\section{ACKNOWLEDGMENTS}

Vladimir Parpura acknowledges the support of this work by a National Science Foundation Award CBET 0943343; he thanks Manoj K. Gottipati for constructive comments on the manuscript. Shivendra Tewari acknowledges useful comments on a previous version of the article by Drs. Kaushik Majumdar and Catherine Kaczorowski.

\section{SUPPLEMENTARY MATERIAL}

The Supplementary Material for this article can be found online at: http://www.frontiersin.org/journal/10.3389/fncom.2013.00145/ abstract 


\section{REFERENCES}

Andersen, P., Morris, R., Amaral, D., Bliss, T., and O'keefe, J. (2007). The Hippocampus Book. New York, NY: Oxford University Press.

Auzmendi, J., Gonzalez, N., and Girardi, E. (2009). The NMDAR subunit NR2B expression is modified in hippocampus after repetitive seizures. Neurochem. Res. 34, 819-826. doi: 10.1007/s11064-008-9828-0

Bushong, E. A., Martone, M. E., and Ellisman, M. H. (2004). Maturation of astrocyte morphology and the establishment of astrocyte domains during postnatal hippocampal development. Int. J. Dev. Neurosci. 22, 73-86. doi: 10.1016/j.ijdevneu.2003.12.008

Bushong, E. A., Martone, M. E., Jones, Y. Z., and Ellisman, M. H. (2002). Protoplasmic astrocytes in CA1 stratum radiatum occupy separate anatomical domains. J. Neurosci. 22, 183-192.

Butler, T. R., Self, R. L., Smith, K. J., Sharrett-Field, L. J., Berry, J. N., Littleton, J. M., et al. (2010). Selective vulnerability of hippocampal cornu ammonis 1 pyramidal cells to excitotoxic insult is associated with the expression of polyamine-sensitive N-methyl-Dasparate-type glutamate receptors. Neuroscience 165, 525-534. doi: 10.1016/j.neuroscience.2009.10.018

Corkin, S. (2002). What's new with the amnesic patient H.M. Nat. Rev. Neurosci. 3, 153-160. doi: 10.1038/nrn726

Danbolt, N. C. (2001). Glutamate uptake. Prog. Neurobiol. 65, 1-105. doi: 10.1016/S0301-0082(00)00067-8

Destexhe, A., Mainen, Z. F., and Sejnowski, T. J. (1994). Synthesis of models for excitable membranes, synaptic transmission and neuromodulation using a common kinetic formalism. J. Comput. Neurosci. 1, 195-230. doi: 10.1007/BF00961734

Ding, S., Fellin, T., Zhu, Y., Lee, S. Y., Auberson, Y. P., Meaney, D. F., et al. (2007). Enhanced astrocytic Ca2+ signals contribute to neuronal excitotoxicity after status epilepticus. J. Neurosci. 27, 10674-10684. doi: 10.1523/JNEUROSCI.2001-07.2007

Eldridge, L. L., Knowlton, B. J., Furmanski, C. S., Bookheimer, S. Y., and Engel, S. A. (2000). Remembering episodes: a selective role for the hippocampus during retrieval. Nat. Neurosci. 3, 1149-1152. doi: 10.1038/80671

Fellin, T., Pascual, O., Gobbo, S., Pozzan, T., Haydon, P. G., and
Carmignoto, G. (2004). Neuronal synchrony mediated by astrocytic glutamate through activation of extrasynaptic NMDA receptors. Neuron 43, 729-743. doi: 10.1016/j.neuron.2004.08.011

Fellin, T., Pascual, O., and Haydon, P. G. (2006). Astrocytes coordinate synaptic networks: balanced excitation and inhibition. Physiology 21, 208-215. doi: 10.1152/physiol.00161.2005

Gasparini, S., Migliore, M., and Magee, J. C. (2004). On the initiation and propagation of dendritic spikes in CA1 pyramidal neurons. J. Neurosci. 24, 11046-11056. doi: 10.1523/JNEUROSCI.2520-04.2004

Gold, C., Henze, D. A., Koch, C., and Buzsaki, G. (2006). On the origin of the extracellular action potential waveform: a modeling study. J. Neurophysiol. 95, 3113-3128. doi: 10.1152/jn.00979.2005

Grover, L. M., Kim, E., Cooke, J. D., and Holmes, W. R. (2009). LTP in hippocampal area CA1 is induced by burst stimulation over a broad frequency range centered around delta. Learn. Mem. 16, 69-81. doi: $10.1101 / \mathrm{lm} .1179109$

Hall, J., Thomas, K. L., and Everitt, B. J. (2001). Cellular imaging of zif268 expression in the hippocampus and amygdala during contextual and cued fear memory retrieval: selective activation of hippocampal CA1 neurons during the recall of contextual memories. J. Neurosci. 21, 2186-2193.

Henneberger, C., Papouin, T., Oliet, S. H., and Rusakov, D. A. (2010). Long-term potentiation depends on release of $\mathrm{D}$-serine from astrocytes. Nature 463, 232-236. doi: 10.1038/ nature 08673

Hirrlinger, J., Hülsmann, S., and Kirchhoff, F. (2004). Astroglial processes show spontaneous motility at active synaptic terminals in situ. Eur. J. Neurosci. 20, 2235-2239. doi: 10.1111/j.1460-9568.2004.03689.x

Holt, W., and Maren, S. (1999). Muscimol inactivation of the dorsal hippocampus impairs contextual retrieval of fear memory. J. Neurosci. 19, 9054-9062.

Kang, N., Xu, J., Xu, Q., Nedergaard, M., and Kang, J. (2005). Astrocytic glutamate release-induced transient depolarization and epileptiform discharges in hippocampal CA1 pyramidal neurons. J. Neurophysiol. 94, 4121-4130. doi: 10.1152/jn.00448.2005

Khuchua, Z., Wozniak, D. F., Bardgett, M. E., Yue, Z., McDonald, M., and Boero, J. (2003). Deletion of the N-terminus of Murine MAP2 by gene targeting disrupts Hippocampal CA1 neuron architecture and alters Contextual Memory. Neuroscience 119, 101-111. doi: 10.1016/S0306-4522(03)00094-0

Knyazev, G. G. (2012). EEG delta oscillations as a correlate of basic homeostatic and motivational processes. Neurosci. Biobehav. Rev. 36, 677-695. doi: 10.1016/j.neubiorev.2011.10.002

Kolarova, A., Ringer, R., Tauber, M. G., and Leib, S. L. (2003). Blockade of NMDA receptor subtype NR2B prevents seizures but not apoptosis of dentate gyrus neurons in bacterial meningitis in infant rats. $B M C$ Neurosci. 4:21. doi: 10.1186/14712202-4-21

Lakatos, P., Karmos, G., Mehta, A D., Ulbert, I., and Schroeder, C. E. (2008). Entrainment of neuronal oscillations as a mechanism of attentional selection. Science 320, 110-113. doi: 10.1126/science. 1154735

Lavialle, M., Aumann, G., Anlauf, E. Prols, F., Arpin, M., and Derouiche, A. (2011). Structural plasticity of perisynaptic astrocyte processes involves ezrin and metabotropic glutamate receptors. Proc. Natl. Acad. Sci. U.S.A. 108, 12915-12919. doi: 10.1073/pnas.1100957108

Lee, I., and Kesner, R. P. (2004). Differential contributions of dorsal hippocampal subregions to memory acquisition and retrieval in contextual fear-conditioning. Hippocampus 14, 301-310. doi: 10.1002/hipo.10177

Liao, G. Y., Wagner, D. A., Hsu, M. H., and Leonard, J. P. (2001). Evidence for direct protein kinase-C mediated modulation of $\mathrm{N}$-methylD-aspartate receptor current. Mol. Pharmacol. 59, 960-964.

Lopez-Aguado, L., Ibarz, J. M., and Herreras, O. (2001). Activitydependent changes of tissue resistivity in the CAl region in vivo are layer-specific: modulation of evoked potentials. Neuroscience 108, 249-262. doi: 10.1016/S0306-4522(01)00417-1

Maren, S. (2001). Neurobiology of pavlovian fear conditioning. Annu. Rev. Neurosci. 24, 897-931. doi: 10.1146/annurev.neuro.24.1.897

Maren, S., and Holt, W. (2000). The hippocampus and contextual memory retrieval in Pavlovian conditioning. Behav. Brain Res. 110, 97-108. doi: 10.1016/S0166-4328(99)00188-6

Martin, E. D., Fernandez, M., Perea, G., Pascual, O., Haydon, P. G., Araque, A., et al. (2007). Adenosine released by astrocytes contributes to hypoxia-induced modulation of synaptic transmission. Glia 55, 36-45. doi: 10.1002/glia.20431

Milner, B., and Penfield, W. (1955). The effect of hippocampal lesions on recent memory. Trans. Am. Neurol. Assoc. $80^{\text {th }} \mathrm{mtg}, 42-48$.

Murchison, C. F., Schutsky, K., Jin, S.-H., and Thomas, S. A. (2011). Norepinephrine and B1-adrenergic signaling facilitate activation of hippocampal CAl pyramidal neurons during contextual memory retrieval. Neuroscience 181, 109-116. doi: 10.1016/j. neuroscience.2011.02.049

Nadkarni, S., and Jung, P. (2003). Spontaneous oscillations of dressed neurons: a new mechanism for epilepsy. Phys. Rev. Lett. 91, 268101. doi: $\quad$ 10.1103/PhysRevLett.91. 268101

Nakazawa, K., McHugh, T. J., Wilson, M. A., and Tonegawa, S. (2004). NMDA receptors, place cells and hippocampal spatial memory. Nat. Rev. Neurosci. 5, 361-372. doi: 10.1038/nrn 1385

Nedergaard, M., Ransom, B., and Goldman, S. A. (2003). New roles for astrocytes: redefining the functional architecture of the brain. Trends Neurosci. 26, 523-530. doi: 10.1016/j.tins.2003. 08.008

Oberheim, N. A., Takano, T., Han, X., He, W., Lin, J. H., Wang, F., et al. (2009). Uniquely hominid features of adult human astrocytes. J. Neurosci. 29, 3276-3287. doi: 10.1523/JNEUROSCI.4707-08. 2009

Ogata, K., and Kosaka, T. (2002). Structural and quantitative analysis of astrocytes in the mouse hippocampus. Neuroscience 113, 221-233. doi: 10.1016/S0306-4522 (02)00041-6

Omkumar, R. V., Kiely, M. J., Rosenstein, A. J., Min, K. T., and Kennedy, M. B. (1996). Identification of a phosphorylation site for calcium/calmodulin dependent protein kinase II in the NR2B subunit of the Nmethyl-D-aspartate receptor. J. Biol. Chem. 271, 31670-31678. doi: 10.1074/jbc.271.49.31670

Paller, K. A., and McCarthy, G. (2002). Field potentials in the human hippocampus during the encoding and recognition of visual stimuli. Hippocampus 12, 415-420. doi: 10.1002/hipo.10053

Parpura, V., and Haydon, P. G. (2000). Physiological astrocytic calcium levels stimulate glutamate release to modulate 
adjacent neurons. Proc. Natl. Acad. Sci. U.S.A. 97, 8629-8634. doi: 10.1073/pnas.97.15.8629

Perea, G., and Araque, A. (2005). Properties of synaptically evoked astrocyte calcium signal reveal synaptic information processing by astrocytes. J. Neurosci. 25, 2192-2203. doi: 10.1523/JNEUROSCI.3965-04.2005

Ruch, S., Markes, O., Duss, S. B., Oppliger, D., Reber, T. P., Koenig, T., et al. (2012). Sleep stage II contributes to the consolidation of declarative memories. Neuropsychologia 50, 2389-2396. doi: $\quad 10.1016 /$ j.neuropsychologia. 2012.06.008

Scoville, W. B., and Milner, B. (1957). Loss of recent memory after bilateral hippocampal lesions. J. Neurol. Neurosurg. Psychiatry 20, 11-21. doi: 10.1136/jnnp.20.1.11

Siekmeier, P. J., Hasselmo, M. E., Howard, M. W., and Coyle, J. (2007). Modeling of contextdependent retrieval in hippocampal region CA1: implications for cognitive function in schizophrenia. Schizophr. Res. 89, 177-190. doi: 10.1016/j.schres.2006.08.007

Small, S. A., Nava, A. S., Perera, G. M., Delapaz, R., and Mayeux,
R. (2001). Circuit mechanisms underlying memory encoding and retrieval in the long axis of the hippocampal formation. Nat. Neurosci. 4, 442-449. doi: 10.1038/ 86115

Takahashi, S., and Sakurai, Y. (2009). Sub-millisecond fi ring synchrony of closely neighboring pyramidal neurons in hippocampal CA1 of rats during delayed non-matching to sample task. Front. Neural Circuits 3:9. doi: 10.3389/neuro.04. 009.2009

Tewari, S., and Majumdar, K. (2012a). A mathematical model for astrocytes mediated LTP at single hippocampal synapses. J. Comput. Neurosci. 33, 341-370. doi: 10.1007/s10827-012-0389-5

Tewari, S. G., and Majumdar, K. K. (2012b). A mathematical model of the tripartite synapse: astrocyte-induced synaptic plasticity. J. Biol. Phys. 38, 465-496. doi: 10.1007/s10867-012-9267-7

Tian, G. F., Azmi, H., Takano, T., $\mathrm{Xu}$, Q., Peng, W., Lin, J., et al. (2005). An astrocytic basis of epilepsy. Nat. Med. 11, 973-981. doi: $10.1038 / \mathrm{nm} 1277$

Traub, R. D., Jefferys, J. G., Miles, R., Whittington, M. A., and Toth,
K. (1994). A branching dendritic model of a rodent CA3 pyramidal neurone. J. Physiol. 481(Pt 1), 79-95.

Traub, R. D., Wong, R. K., Miles, R., and Michelson, H. (1991). A model of a CA3 hippocampal pyramidal neuron incorporating voltage-clamp data on intrinsic conductances. J. Neurophysiol. 66 635-650.

Zhang, X.-H., Wu, L.-J., Gong, B., Ren, M., Li, B.-M., and Zhuo, M. (2008). Induction- and conditioningprotocol dependent involvement of NR2B-containing NMDA receptors in synaptic potentiation and contextual fear memory in the hippocampal CA1 region of rats. Mol. Brain 1:9. doi: 10.1186/17566606-1-9

Zhao, M.-G., Toyoda, H., Lee, Y.-S., Wu, L.-J., Ko, S. W., Zhang, X.-H., et al. (2005). Roles of NMDA NR2B subtype receptor in prefrontal long-term potentiation and contextual fear memory. Neuron 47, 859-872. doi: 10.1016/j.neuron. 2005.08.014

Zhuo, M. (2009). Plasticity of NMDA receptor NR2B subunit in memory and chronic pain. Mol. Brain 2, 4 doi: 10.1186/1756-6606-2-4
Conflict of Interest Statement: The authors declare that the research was conducted in the absence of any commercial or financial relationships that could be construed as a potential conflict of interest.

Received: 09 July 2013; accepted: 03 October 2013; published online: 05 November 2013.

Citation: Tewari S and Parpura V (2013) A possible role of astrocytes in contextual memory retrieval: An analysis obtained using a quantitative framework. Front. Comput. Neurosci. 7:145. doi: 10.3389/ fncom.2013.00145

This article was submitted to the journal Frontiers in Computational Neuroscience.

Copyright (c) 2013 Tewari and Parpura. This is an open-access article distributed under the terms of the Creative Commons Attribution License (CC BY).

The use, distribution or reproduction in other forums is permitted, provided the original author(s) or licensor are credited and that the original publication in this journal is cited, in accordance with accepted academic practice. No use, distribution or reproduction is permitted which does not comply with these terms. 